\title{
LARGE HIGHLY POWERFUL NUMBERS ARE CUBEFUL
}

\author{
C.B. LACAMPAGNE* AND J.L. SELFRIDGE
}

Let the prodex of $n$ be the product of the exponents of the primes when $n$ is written in standard form. M. V. Subbarao has called a number highly powerful if its prodex is larger than that of any smaller number. Assume that $n=\prod_{i=1}^{k} p_{i}^{E}$ is highly powerful. Then it is clear that $p_{i}$ is the $i$ th prime, the exponents $E=E\left(p_{i}\right)$ are nonincreasing, $E\left(p_{k}\right) \geqq 2$ and $E\left(p_{k-1}\right)$ $\geqq 3$ (since $\left.p_{k-1}^{4}<p_{k-1}^{2} p_{k}^{2}\right)$. The theorem of the title asserts that if $p_{k}>N$, then $E\left(p_{k}\right) \geqq 3$. Further, we have developed an algorithm which finds all highly powerful numbers having $E\left(p_{k}\right) \neq 3$. The nineteen highly powerful numbers with $E\left(p_{k}\right)=2$ are listed in Table 1 .

Table 1

THE 19 HIGHLY POWERFUL NUMBERS WHICH ARE NOT CUBEFUL

\begin{tabular}{lll}
\hline $2^{2}$ & $2^{8} 3^{4} 5^{2}$ & $2^{11} 3^{6} 5^{5} 7^{4} 11^{3} 13^{3} 17^{2}$ \\
$2^{4} 3^{2}$ & $2^{7} 3^{5} 5^{3} 7^{2}$ & $2^{10} 3^{7} 5^{5} 7^{4} 11^{3} 13^{3} 17^{2}$ \\
$2^{5} 3^{2}$ & $2^{7} 3^{4} 5^{4} 7^{2}$ & $2^{11} 3^{7} 5^{5} 7^{4} 11^{3} 13^{3} 17^{2}$ \\
$2^{7} 3^{3} 5^{2}$ & $2^{8} 3^{5} 5^{3} 7^{2}$ & $2^{11} 3^{7} 5^{5} 7^{4} 11^{3} 13^{3} 17^{3} 19^{2}$ \\
$2^{6} 3^{4} 5^{2}$ & $2^{8} 3^{4} 5^{4} 7^{2}$ & $2^{11} 3^{8} 5^{5} 7^{4} 11^{3} 13^{3} 17^{3} 19^{2}$ \\
$2^{5} 3^{5} 5^{2}$ & $2^{9} 3^{6} 5^{4} 7^{3} 11^{2}$ & \\
$2^{7} 3^{4} 5^{2}$ & $2^{11} 3^{7} 5^{4} 7^{3} 11^{3} 13^{2}$ & \\
\hline
\end{tabular}

\section{REFERENCES}

C. B. Lacampagne and J.L. Selfridge, Large Highly Powerful Numbers are Cubuful, Proc. Amer. Math. Soc. 91 (1984), 173-181.

University of Michigan, Flint, Michigan.

Received by the editors on May 11, 1983.

Copyright (C) 1985 Rocky Mountain Mathematics Consortium 
